\title{
DEVELOPMENT OF UNDERWATER ACOUSTIC MODEMS AND NETWORKS
}

\author{
By Josko Catipovic, David Brady, and Steven Etchemendy
}

\section{Applications for}

underwater

communications . . .

focus on real-time

communication . . .
$\mathrm{R}$ communication enable real-time contact with underwater sensors, vehicles, and other instruments, offering novel ocean observational capabilities that may change future oceanographic operations. This article summarizes the current state of the art in underwater acoustic communication and telemetry equipment. Acoustic transmission is an attractive option for underwater communication only because radio frequency (RF) and optical energy are severely attenuated under water, whereas acoustic energy propagates well. Information encoded and transmitted on a modulated acoustic waveform can be received and decoded by distant underwater receivers.

The relatively slow speed of sound (compared with the speed of light) causes extensive multipath fluctuation and Doppler shifts of the received waveforms. The relative complexity of the oceanic waveguide causes strong spatial, temporal, and frequency-dependent fluctuation. These effects are much more pronounced than those in RF propagation and require rather different approaches to system design. Historically, straightforward "grafts" of RF communication methods to the ocean environment have met with poor success.

Underwater communication started with the underwater telephone or "Gertrude" shortly after WWII. Its derivatives are in common use today, primarily for voice communication between divers and with submersibles. In the 1960 s there were several attempts at digital communication (Baggeroer, 1984). By and large, communication technology of the time was inadequate for a waveguide as complex as the underwater channel. In the 1970 s and 1980s digital microprocessor tcchnology became available and turned out to be the key enabling technology for underwater modems. Several

J. Catipovic, Woods Hole Oceanographic Institution. Woods Hole, MA 02543, USA; D. Brady, Northeastern University, Boston. MA 02115. USA; and S. Elchemendy. Monterey Bay Aquarium Research Institute, Pacific Grove, CA 93950. USA. systems were built and operated in the water (Catipovic. 1990). However, the cost of implementation at that time precluded commercialization of most high-rate systems. The U.S. Navy apparently used several low-rate long-range classified systems at this time. A summary of the state of the art in the field in 1984 appears in IEEE Journal of Oceanographic Engineering (1984).

The explosion of low-cost digital signal processing (DSP) hardware in the late 1980s, along with parallel advances in RF and telephone communications, stimulated research in this area. It became possible to build entire acoustic modems on a few integrated circuits, and interest in commercially viable underwater modems increased. Several high-rate and long range prototypes were built and were successfully commercialized (Catipovic et al., 1990).

Recent advances in adaptive equalization (Proakis, 1983) have pointed the way for greatly increased data rates through the use of signal phase as an information carrier. The resultant "phase-coherent" systems are more efficient in bandwidth and power requirements, but require much more elaborate channel estimation and equalization algorithms (Stojanovic et al., 1993).

Applications for underwater communications currently focus on real-time communication with remote instrumentation and equipment. This has led to development of undersea communication networks that attempt to bring the convenience of cellular communications to remote ocean-bottom regions (Curtin et al., 1993, this issue). The goal of ocean network developments is to permit autonomous cellular communication between landbascd nctworks. such as Internet, and remote instrumentation deployed on the ocean bottom. Instrumentation equipped with cellular acoustic modems can communicate with labs without hardwired cables or dedicated surface moorings. We expect that providing cellular communications coverage in high-interest oceanic areas has the potential to affect our oceanic observational capability significantly. 


\section{Acoustic Modem Performance Regions}

Before discussing the details of current acoustic modems, a brief presentation of performance limitations in acoustic communication is in order. Three distinct performance regions have emerged from acoustic propagation, signal processing, and application concerns. Current work is addressing both point-to-point and networked communication in all three areas:

1. Long-range systems $(20-2,000-\mathrm{km}$ range $)$ in deep water have an upper frequency limit between $500 \mathrm{~Hz}$ and $10 \mathrm{kHz}$. High attenuation at long ranges requires transmitter power levels in excess of 100 watts. The long range acoustic channel has a rather long multipath spread, but the channel phase stability is rather high, and phase-coherent methods are generally used in this regime. Generally, the main problem is transmitting sufficient power to overcome the high transmission loss. The long-range acoustic channel is a very complex waveguide; the high spatial transmission loss (TL) variability requires either that the system be operated in regions where the channel fluctuations and spatial dependence are low, or that real-time channel modeling be integrated into system operation to avoid acoustic "shadow zones", i.e., regions of very high TL. Recent developments in multichannel communication receivers somewhat alleviate the shadow zone problem, because only some of the receiver hydrophones need to be located in regions of acceptable performance (Stojanovic et al., 1993). Typical data rates currently achievable at long ranges are $100 \mathrm{bits} / \mathrm{sec}$ at $1 \mathrm{Mm}$ to $10,000 \mathrm{bits} / \mathrm{sec}$ at $20 \mathrm{~km}$.

2. Medium-range systems operate in the range of $1-10 \mathrm{~km}$ and are often required to operate in shallow water and in the presence of extended time-varying multipath. The upper frequency limit ranges from 10 to $100 \mathrm{kHz}$. The channel dynamics are high. Medium-range systems are often asked to operate with high source/receiver velocities in enclosed areas with extensive multipath structures where the line-of-sight path does not constitute the principal arrival and in the presence of high noise levels, such as may be caused by nearby ship traffic or the bustle at a marine worksite. The primary performance limitation is the systems' ability to compensate for the channel dynamics. Data rates of $10-50 \mathrm{kbits} / \mathrm{sec}$ are achievable at these ranges.

3. Short range systems, operating at ranges of $<1 \mathrm{~km}$ and in deep water are characterized by relatively mild phase fluctuations and somewhat larger bandwidth availability. These channels are amenable to many communication methods developed for digital telephony and RF telemetry. Relatively simple implementations have been shown to yield data rates from 10 to $>100 \mathrm{kbits} / \mathrm{sec}$. At ranges of $<100 \mathrm{~m}$ in relatively clear water, laseroptic communication is feasible at much higher data rates.

\section{WHOI/MIT Acoustic Modem Development \\ Program}

The program was started by Arthur Baggeroer in 1978, partially motivated by a contemporary publication by Pieper et al. (1979; this publication reported partial results of classified work in longrange undersea communications conducted by Pieper et al. on behalf of Sperry.) The objective of the work was to understand digital communication over extremely dynamic channels and to demonstrate an all-digital system in the shallow-water environment. The resultant digital acoustic telemetry system (DATS) was a direct precursor of the current generation of underwater modems (Baggeroer et al., 1981). The decision to use DATS as a testbed of new technology and state-of-the-art communication engineering was far-reaching. Because the goal of the effort was not to build an operationally useful system, the program was free to explore novel methods and advanced, yet unproven, technology. (Catipovic et al., 1984)

The DATS system was portable and was extensively tested in Woods Hole harbor. The original goal of the project was to study communications over rapidly variant channels, and the Woods Hole harbor test site provided extended and highly dynamic multipath conditions. The system was also used under ice in Lake Caanan. New Hampshire, and in the Marginal Ice Zone near Svalbard (Hanot, 1980). In 1984 DATS was retired because of physical wear.

The next step in the program was to build a less complex, inexpensive acoustic modem that could be used for oceanographic data acquisition. We drew on the DATS to develop a more compact version using the newly available DSP technology. The effort was structured into three stages (Catipovic and Freitag, 1990):

1. Validation of theoretical results was accomplished by recording underwater acoustic data transmissions and developing receiver and processing algorithms off-line on prestored data. This allowed simple developing and testing of advanced processing solutions without committing to efficient receiver design. Candidate techniques were compared and several receiver realizations selected for real-time implementations.

2. A prototype system based on Inmos Transputers was used as a testbed for developing and evaluating receiver algorithms in real-time (Fig. 1). Some of the implementation results are reported in (Catipovic and Baggeroer, 1990) and (Catipovic and Freitag, 1991). The real-time prototype was tested extensively in Woods Hole harbor and in deep water. The principal goal was to find an algorithm suitable for an inexpensive, robust modem for oceanographic use.

3. A 16-tone multiple-frequency shift keying (MFSK) receiver algorithm was selected for commercialization. At this stage we approached sev-
The objective was to

understand digital

communication over

extremely dynamic

channels... . 


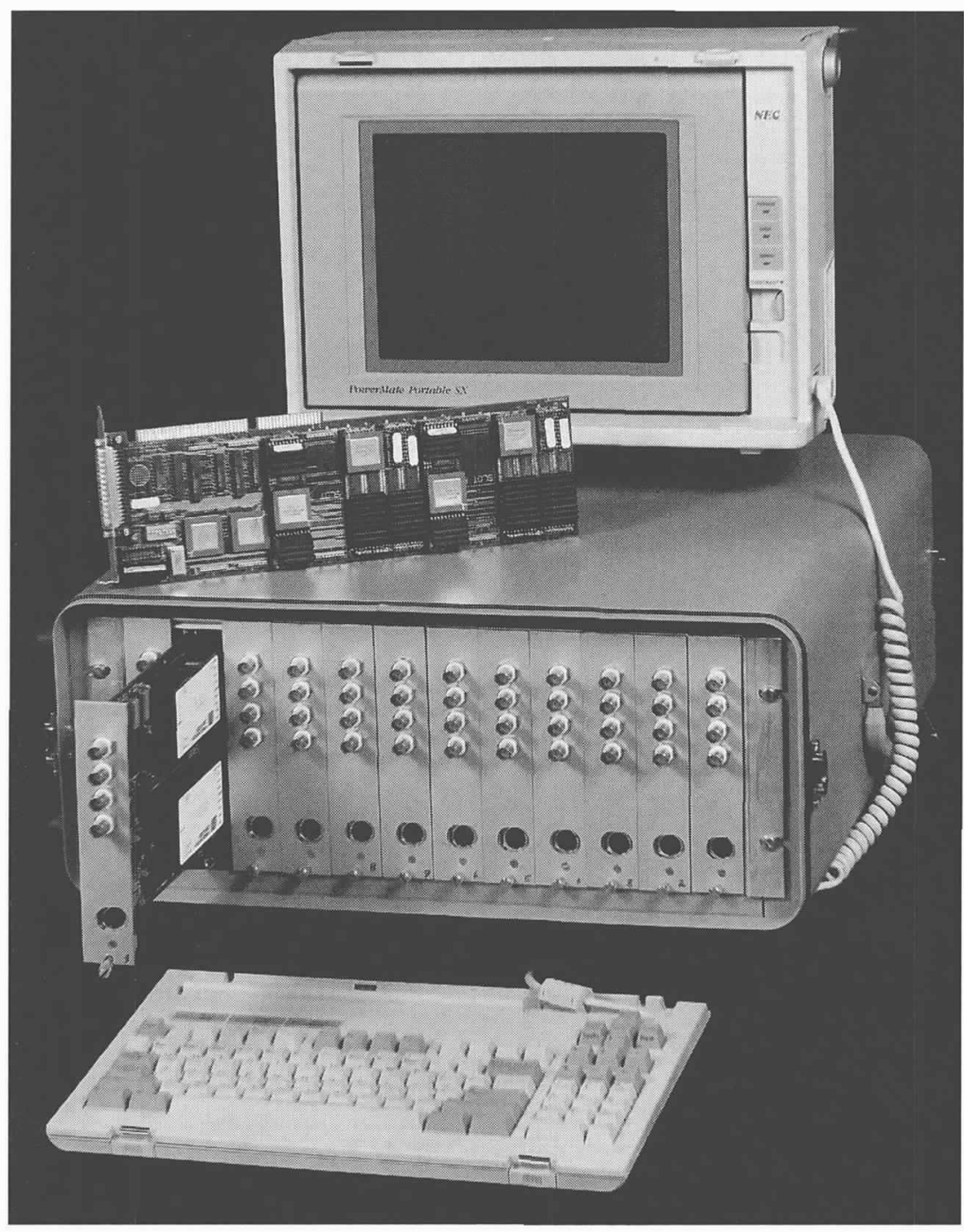

Fig. 1: Prototype acoustic telemetry receiver.

demand for the technology came from the oil industry . . . to replace data cables at offshore platforms. eral firms with a technology transfer proposal. Guessing that the best choice for a technology transfer partner would be a small firm with experience in oceanographic technology, we discussed our ideas in 1987-1988 with Ferrante ORE, Benthos, Seadata, Marquest, RDI, and Datasonics. Only Datasonics was interested in committing to a joint program in developing underwater modems. A joint development program began in 1988.

\section{Technology Transfer Program}

The WHOI-Datasonics collaboration in underwater modem development has led to a line of commercially available modems that are beginning to find acceptance in the marketplace. The division of development effort agreed upon left WHOI with responsibility for prototype development, including algorithm and software development as well as design and construction of the production prototypes. Datasonics responsibilities included acoustic transducers, and mechanical tasks, including packaging of electronics, pressure housings, connectors, batteries, etc. In retrospect, tasks were well divided and construction of initial production units was relatively straightforward and successful.

The design trade-offs in the development of the modems centered on selecting the product that would improve the users' confidence in acoustic communication. The market had seen (and remembered) previous modems, characterized by marginal quality and often being inoperable. The first step required building user confidence and demonstrating that real-time communication was possible, robust, and practical. At this stage, maximizing data rate took a back seat to developing a simple, robust instrument that could build user confidence. At the same time, we took care to design the modems for a performance upgrade path, so that higher data rates, ranges, and power efficiency improvements could be added with minimal hardware modifications.

The modems implemented MFSK modulation in compact packages suitable for full ocean depth deployment. The acoustic telemetry modem (ATM) 845 is shown in Figure 2. It is a powerefficient unit capable of 1,200 bit/sec data transmission. It has an FSK receiver capable of 80 bits/sec. The unit is intended for ocean-bottom instrumentation, where the data flow is primarily from the instrument to the surface, and only occasional commands are transmitted to the instrument. The ATM 845 is packaged in a 26 inch long, 5 inch dia. pressure housing that also contains a battery supply sufficient for transmitting $\sim 20$ Mbytes of data. Standby power drain is $\sim 1 \mathrm{~mW}$, and deployment times in excess of 1 year are practical.

A Datasonics ATM 850 acoustic modem that resulted from this effort is shown in Figure 3. It is a minimal-hardware implementation of the prototype shown in Figure 1. The ATM-850 is capable of transmitting and receiving data at 2,400 bits/sec. It is built around an AT\&T DSP32C processor and is intended for applications requiring fast transmission and reception. It is used as the surface receiver for telemetry from subsurface instruments and also for autonomous underwater vehicles (AUVs), which both transmit and receive large amounts of data. The ATM 850 is fully compatible with the ATM 845. Its power drain is $\sim 5 \mathrm{~W}$ when receiving and $\sim 10 \mathrm{~mW}$ in standby mode. Transmit efficiencies for both units are on the order of 1,000 bits/joule per $\mathrm{km}$.

The first demand for the technology came from the oil industry, eager to replace data cables at offshore platforms. Datasonics delivered a few modems for tasks such as offshore platform status monitoring and communication with pipeline inspection tools ("pipeline pigs," as they are known in the industry). These orders sustained the effort during the early stages of development.

Current work on the ATM-series modems centers around development of interface drivers, im- 


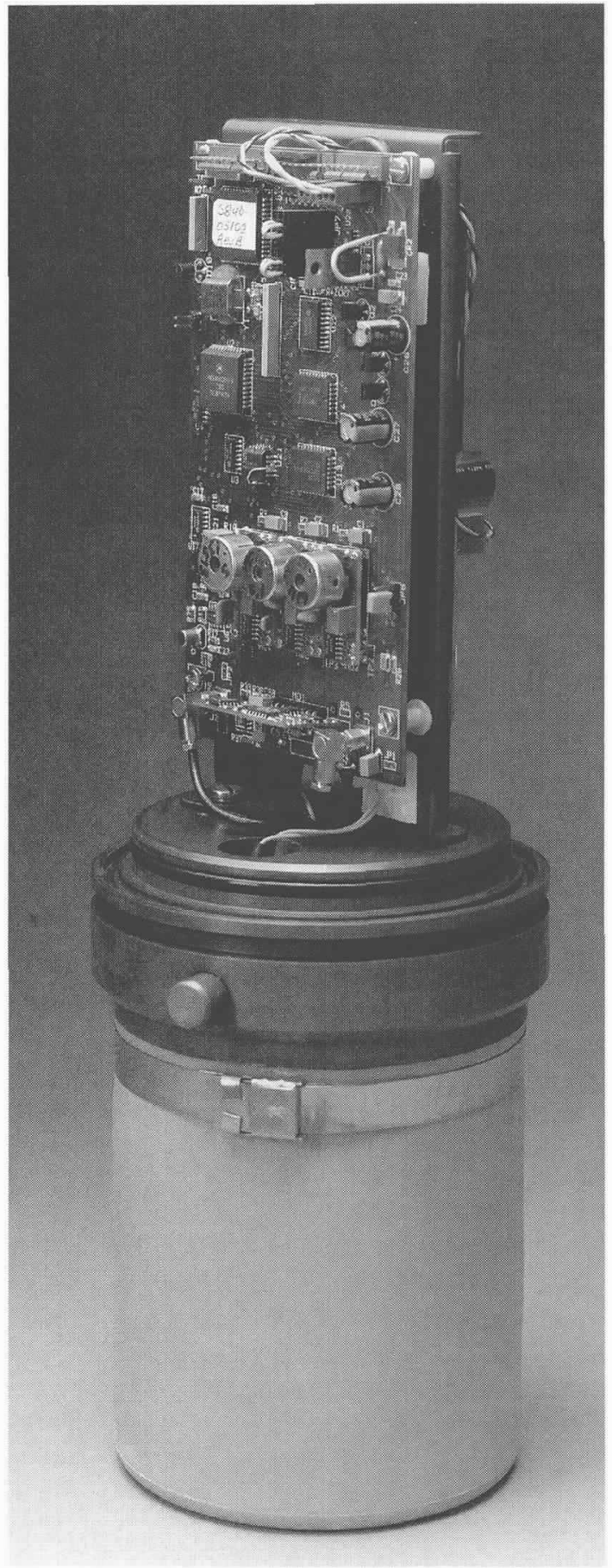

Fig. 2: Datasonics ATM 845 acoustic modem.

provements in data transmission software, and increases in data rate, range, and reliability. A phasecoherent transmission capability for the ATM-series modems is currently undergoing testing during an extended deployment in Monterey canyon. We expect to increase the modems' capabilities gradually while perserving the users' hardware investments.

The technology transfer effort has been a moderately frustrating experience, which serves to illustrate difficulties of moving a novel technology into the restricted oceanographic market. The principal issue was the development of a market for a rather new technology. The Datasonics modems performed a novel function, which was not available to the community earlier, and users had to modify their ongoing efforts to take advantage of the new technology. Before the modems' introduction, there had been a few custom prototypes used for data acquisition, but acoustic telemetry from remote instrumentation was not an option for most oceanographic work. Furthermore, oceanographic data acquisition is an essentially noncompetitive endeavor, with a small number of players conducting long-term programs with rather stable federal funding. Introducing a new technology into this community was a surprisingly difficult and long-term effort because it perturbed status quo and because such programs require long-term planning. Selecting a small firm, was, in retrospect, a good choice. Although a larger

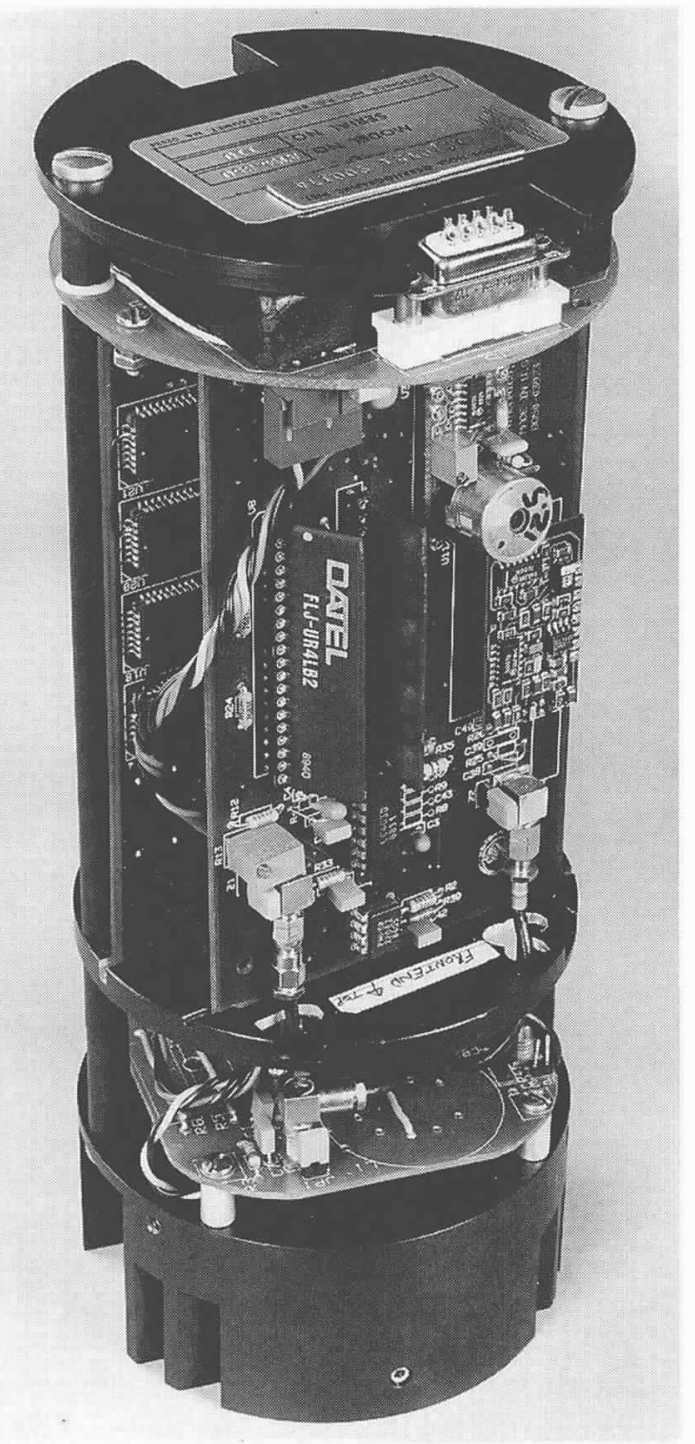

Fig. 3: Datasonics ATM 850 acoustic modem.

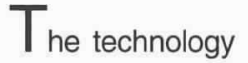

transfer effort has been a moderately frustrating experience, . . . 
firm could have invested more resources in development, the product probably would have cost more, and would not have been accepted into the oceanographic market. The small firm walks a fine line between restricting itself to a few well-known. accepted products and introducing new technologies with lengthy break-in periods. Of the six firms we initially talked to, four saw acoustic modems as too high a risk area for a potential product line.

Currently, the technology transfer is well established. Modem sales are increasing, still led by the offshore oil industry, but the number of scientific users is increasing. Modem cost is dropping while the capabilities and reliability are increasing. Unfortunately, relative success has brought along a new set of difficulties as corporate and investor pressure grows. As research funding is perceived to tighten, academic institutions see technology transfer as an important new source of income. This has placed a new burden on our effort by formalizing the effort and causing it to be perceived as a source of licensing and royalty income. Although these efforts may be successful, they risk smothering fledgling efforts. It is possible that the technology transfer requirements and the recent interest in "economic competitiveness" will cause damage to the very fragile, small, and specialized oceanographic technology market, which developed through informal contacts between researchers and small, local firms.

s research funding

is perceived to tighten,

academic institutions

see technology transfer

as an important new

source of income.
3. A shallow water experiment conducted in Buzzards Bay, MA, in February 1992 demonstrated $20 \mathrm{kbit} / \mathrm{sec}$ communication at $3 \mathrm{nmi}$ and 10 $\mathrm{kbit} / \mathrm{sec}$ at $4 \mathrm{nmi}$. The experiment was conducted in $\sim 10 \mathrm{~m}$ water depth.

These results have encouraged us to build a compact modem with minimal hardware. Modem development is underway with ARPA sponsorship. Much of the capability has also been implemented on the modems shown in Figures 2 and 3.

\section{Acoustic Local and Wide Area Networks}

Most applications for undersea communication call for long-term monitoring of selected ocean areas, such as hot vent areas at midoceanic ridges or ocean dumpsites. The ideal communication solution for these applications is a local area network, emulating the familiar Internet computer network that is used extensively by the research community. However, existing network protocols, typically designed for RF or hard-wired applications, were not compatible with acoustic links.

Several attributes of underwater acoustical telemetry links are quite different from those of terrestrial-based RF systems and render RF-based protocols inefficient or useless for underwater applications. The predominant difference is the ratio of one-way propagation time to packet duration. and this delay has a dramatic impact on the protocol selection. For example, in the two underwater networks deployed to date, this relative propagation delay falls in the range of $1-100$ times the packet size. For terrestrial-based systems with the same geographical coverage (100-1.000 square kilometers) and the same packet length, this propagation delay is five orders of magnitude smaller, due to the ratio of propagation velocities of light and sound. This significant difference makes propagation time an important component in the network delay of underwater systems and suggests a sparing use of handshaking, acknowledgments, and retransmissions between acoustical modems. In addition, the relatively infrequent access of modems to the network renders fixed-time or fixed-frequency assignment schemes inefficient for underwater acoustical schemes, as each subchannel would be poorly utilized. Another consideration is the geographical diversity of the underwater modems. This diversity produces a large variation in one-way propagation time and precludes the use of global synchronization, which is required for all time-sharing or time-slotted protocols.

Another significant difference between landand ocean-based networks is the importance of the modem power sources for the design of protocols. Even though land-based systems are designed with considerations of the received power of a transmitter, very little attention is devoted to the finite energy constraints of battery-powered modems. This is reasonable, considering that batteries are easily replaced on land-based systems. In ocean deployments, however, the replacement 
of a modem battery involves ship time and the retrieval of acquisition systems from the ocean bottom or water column, and frequently terminates the experiment. This difference makes transmission energy precious in underwater applications, and efficient protocols should allow modems to power-down between infrequent transmissions. Retransmissions have a significantly higher cost in ocean networks and are usually avoided.

The acoustic telemetry channel has other features that must be considered in the design of an efficient network protocol. The ocean is a broadcast acoustical channel that occupies a rather narrow frequency band (5-10 times a modem's peak symbol rate), and transmissions often interfere with each other at a receiver. These collisions may not be easily avoided by premature termination of transmission (because of the relatively long propagation delays), the assignment of separate transmission bands or times (because of infrequent transmission of each modem), or polling/probing techniques (because of the delay incurred by handshaking as well as the power-down status of idle modems). Each transmission may also be affected by significant and time-varying distortion due to reverberation in the ocean channel.
The efficient flow of data packets along a changing topology of communication links is the primary goal of a network protocol. Historically, the two measures of protocol efficiency have been network throughput (the average rate of successful packets) as well as the average transmission delay incurred by a packet. Because these figures of merit usually oppose each other, a network designer synthesizes a protocol that strikes a reasonable balance between throughput and expected delay. Because the throughput and average packet delay of any protocol are heavily dependent on the physical properties of both the transmission media and the transceivers, a protocol that is efficient for one scenario may perform quite poorly in another.

\section{The ALAN Protocol}

This section presents an example of a network protocol for an acoustic telemetry system. This particular protocol is used for a deep water network currently deployed in Monterey Canyon, off California. We shall first describe the topology of the network nodes and then detail the structure of the protocol from the perspective of a new packet entering the network. We shall also illustrate how the differences between land- and ocean-based

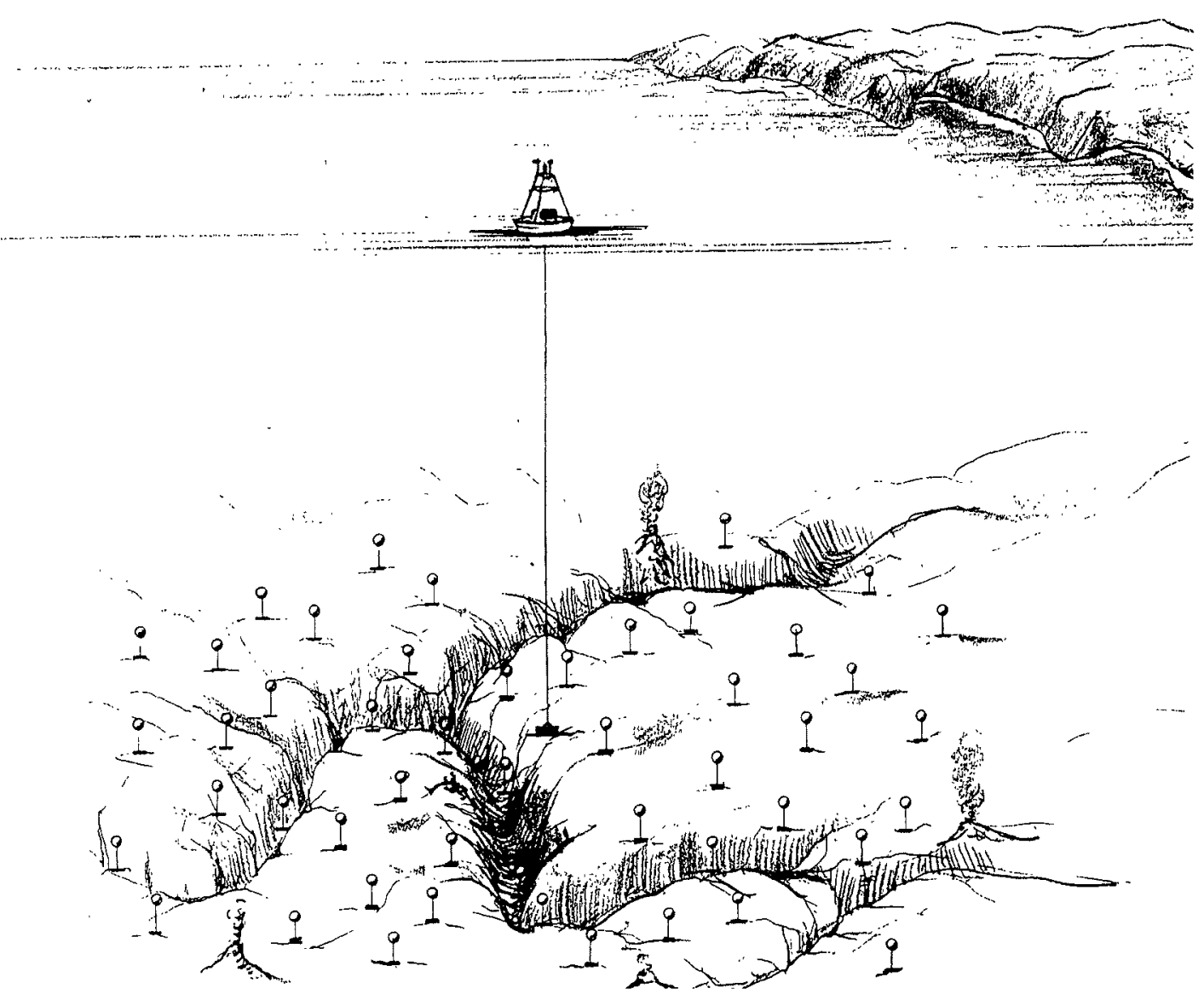

... a protocol that is efficient for one scenario may perform quite poorly in another.

Fig. 4: Acoustic local area network 
systems described in the last section influenced the selection of the protocol.

The configuration of the nodes in the acoustic local area network (ALAN) is depicted in Figure 4. This network is designed to be used for longterm (1-5 years) data acquisition and ocean monitoring from many ocean bottom sources. In this scenario the ocean-bottom nodes are attached to stationary data sources and transmit data packets to a central, surface-deployed receiver. These packets are later transmitted to shore in a batch fashion via an RF link. Although the primary data flow is from the ocean bottom to the central receiver, there is a modest communication link from the shore terminal to any node for interaction with the data source. The exchange of information between the surface receiver and the downlink station on the shore is very similar to an electronic mail system on the Internet. Data from a particular sensor is forwarded to the mailbox of its owner, and interaction with the ocean-bottom sensor is achieved by transmittal of a mail message to the ocean-bottom source, as described below.

A time-frequency diagram is depicted in Figure 5 and illustrates the complete transmission cycle of a packet from a data source on the ocean bottom. An ocean-bottom modem remains in a lowenergy mode until the data source has collected a predetermined amount of information. The modem then awakes and initiates a network session by transmitting a short request packet on a narrow asynchronous channel, which is common to all modems. Figure 5 illustrates the traffic on the request channel due to the fixed length packets, from the time reference of the surface receiver. Packets are labeled in the figure by the modem ID. The request packet contains the requesting

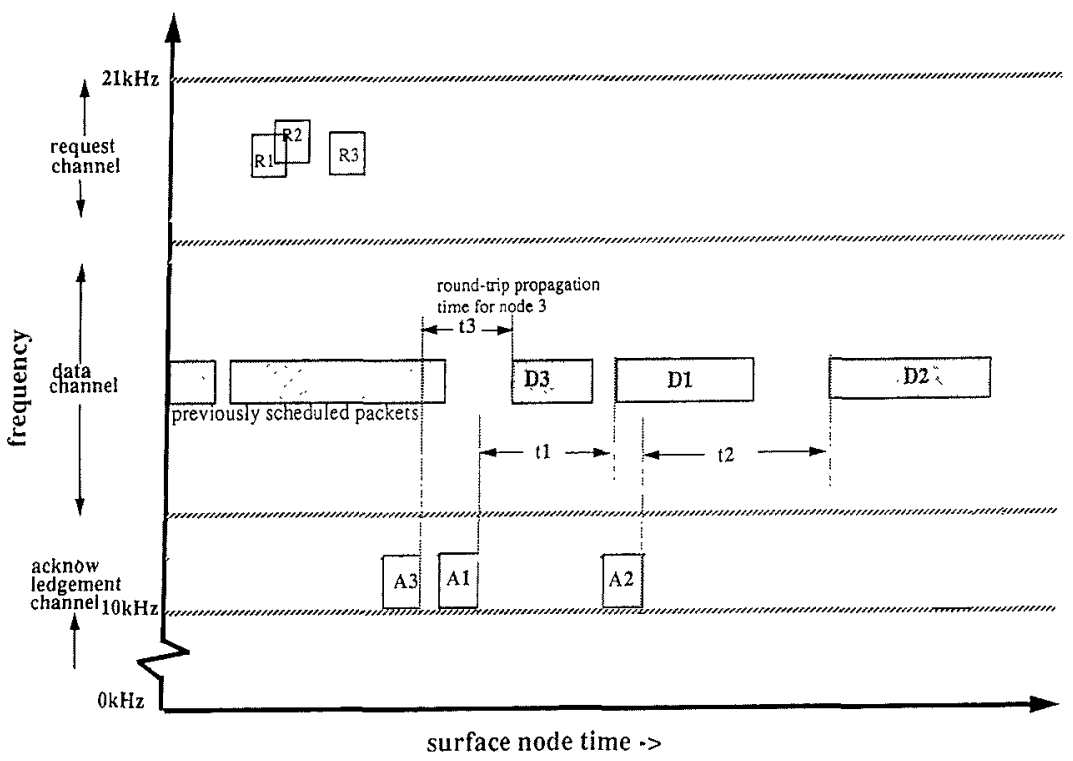

Fig. 5: Acoustic local area network communication protocol. modem ID and size of the buffer that must be transmitted to shore. The ocean-bottom modem then waits for an acknowledgment packet (goahead signal) from the surface receiver. This packet is transmitted over a separate acknowledgment channel. The modem transmits data on the specified data channel, at the moment of reception of the acknowledgment packet. Note that the acknowledgment packets are not transmitted in the order of request packet reception, as shown in Figure 5. The central receiver tracks the round-trip propagation time of each modem and uses this information to determine both the data channel schedules and the acknowledgment schedule. If an acknowledgment packet is not received within a specified time-out period (roughly 20 seconds), then the request process is repeated by the oceanbottom modem. In the example illustrated in Figure 5, there are three channels in the 10- to 21 $\mathrm{kHz}$ frequency range: one data channel, an acknowledgment channel. and a request channel.

The primary tasks of the surface receiver are to demodulate request packets, to schedule the corresponding data sessions, and to send acknowledgments at the appropriate times. Because of the inability to coordinate requests on the common channel, the receiver must contend with the collision of request packets from several modems. $\mathrm{Al}-$ though such collisions are often discarded in landbased systems, the request channel receiver in this network is designed to unscramble the collision of several request packets. This resolution of request packet collisions reduces both the amount of time that an ocean-bottom modem must remain in fullpower mode and the expected packet delay caused by the time-out period. Upon successful reception of a request packet, the requested amount of time is scheduled in one of two data channels (whichever one minimizes the schedule delay). The surface receiver then transmits an acknowledgment packet at a time that precedes the scheduled arrival time by an amount equal to the round-trip propagation delay of the particular modem and notifies the surface data channel receiver at the scheduled arrival time. The surface receiver maintains the timing for the schedule and also updates the round-trip propagation time of the particular ocean-bottom node upon reception of the data packet. Data channel scheduling maintains sufficient guard time between packets to avoid collisions due to errors in the round-trip propagation time.

This protocol attempts to capitalize on the knowledge of the round-trip propagation times of the ocean-bottom nodes and to utilize fully the data channels. Without utilizing the round-trip propagation times, a collision-free data channel schedule would require large time gaps between the data channel packets, corresponding to the maximum round-trip propagation time (roughly 10 seconds). This advantage is eroded in the absence of accurate 
estimates of the round-trip delays (within 50 milliseconds). The problem may be avoided by enforcing a minimum level of activity of all network nodes, corresponding to the anticipated rate of change of the round-trip propagation time: a roving node might be required to send frequent, short data packets, while a moored node might have a very negligible minimum request rate.

The resultant network is currently deployed in Monterey Canyon, as part of a collaborative effort between Woods Hole Oceanographic Institute, Northeastern University, and Monterey Bay Aquarium Research Institute (MBARI). Our goal is to demonstrate long-term real-time communication with numerous ocean-bottom sensors. We expect the network to remain deployed in the foreseeable future and to encourage prospective users to participate with their ocean-bottom equipment. To date, the network includes U.S. Geological Survey ocean-bottom seismometers, a chemical osmotic pump, current meters, CTDs. and temperature sensors. We have comittments to include ocean-bottom hydrophones, vertical acoustic arrays, CCD cameras, and AUVs. MIT's AUV Odyssey is currently equipped with an acoustic modem and is undergoing trials to quantify modem performance at high speeds.

The immediate goal of the Monterey Canyon ALAN is to interface ocean-bottom instruments directly into the Internet network. We hope to show to the scientific community the usefulness of real-time contact between an office workstation and remote deployed equipment. The plan is to build user confidence with the system deployed in Monterey, and at the same time to pursue a second deployment, most likely in the Juan de Fuca area. A shallow water network is scheduled for 1994 deployment on the south New England continental shelf, and an under-ice network deployment is scheduled for spring of 1994 (Curtin et al., 1993, this issue).

\section{Acknowledgements}

The ALAN project is supported by the National Science Foundation, Oceanographic Technology Division, grant OCE-92-01191, by the Monterey Bay Aquarium Research Institute, and by Datasonics, Inc.

\section{References}

Baggeroer. A.B.. 1984: Acoustic telemetry-an overview. IEEE J. Ocean. Engin.. OE-9, 229-234.

Baggeroer, A.B., D. Koelsch. K. von der Heydt, and J. Catipovic. 1981: DATS - a digital acoustic telemetry system for underwater communications. Proceedings of the Oceans ' 81 Conference, 1981, Boston. MA. 55-60.

Catipovic. J.. 1990: Performance limitations in acoustic telemetry. IEEE J. Ocean. Engin., 15, 205-216.

Catipovic. J. and A.B. Baggeroer, 1990: Analysis of high frequency multitone transmissions propagated in the marginal ice zone. J. Acoustic. Soc. Am. 88, 185-190.

Catipovic. J. and L. Freitag, 1991: Spatial diversity processing for underwater acoustic telemetry. IEEE J. Ocean. Eng.. 16. 86-97.

Catipovic. J.. D. Frye. and D. Porta, 1990: Compact digital signal processing enhances acoustic data telemetry". Sea Technology, May 1990. pp 10-15.

Catipovic. J.. K. von der Heydt, J.S. Merriam, and G.H. Sandsmark. 1991: Multiple convergence zone acoustic telemetry feasibility test report. WHOT Technical Report, WHOI-91-38

Curtin. T.B., J.G. Bellingham, J. Catipovic, and D. Webb, 1993: Autonomous oceanographic sampling networks. Oceanography, 6, 86-93.

Hanot, W., 1980: A phased array sonar for an underwater acoustic communication system. M.S. thesis. MIT Dept. of Ocean Engineering.

IEEE Journal of Oceanographic Engineering special issue on acoustic telemetry, OE-9 October 1984.

Pieper, J.E., J.A. Proakis, R.R. Reed, and J.K. Wolf, 1978: Design of efficient coding and modulation for a Rayleigh fading channel. IEEE Trans. Inform. Theory, IT-24, $457-468$.

Proakis, J., 1983: Digital Communications, 2nd ed. McGraw Hill. New York, 905pp.

Stojanovic, M.. J. Catipovic. and J. Proakis, 1993: Adaptive multichannel combining and equalization for underwater acoustic communication. J. Acoustic. Soc. Am. 9.4. $1621-1631$

Catipovic. J.A.. A.B. Baggeroer, K. von der Heydt, and D. Koelsch. 1984: Design and performance analysis of a digital acoustic telemetry system. IEEE J. Ocean. Eng., $O E-9.242-252$.

Catipovic, J. and L. Freitag. 1990: High data rate acoustic telemetry for moving ROV-s in fading multipath shallow water environments. Proceedings of the 7th IEEE International Symposium on Unmanned Untethered Submersible Technology, Washington, D.C., June 1990, 296-303.

Schmidt. H. et al. 1993: Under-ice mapping with an autonomous underwater vehicle. Proceedings of the Oceans 93 Conference, 1993. Victoria, Canada. $\square$ 